\title{
Floral diversity assessment of the buffer zones and vicinity of the Mt. Hamiguitan Range Wildlife Sanctuary (MHRWS), Davao Oriental: basis for inclusion to protected area zone
}

\author{
Victor B. Amoroso*, Florfe M. Acma, Fulgent P. Coritico, Felipe S. Gorme, Noel E. Lagunday, Mary Cor S. Salolog, \\ and Ruel D. Colong
}

\begin{abstract}
In 2016, municipal ordinances to expand the protected area of the MHRWS were issued with the aim of protecting and preserving the remaining biodiversity of the buffer zones and to strengthen the core zone. The municipal ordinances however, have limitations and do not guarantee legal promulgation. Hence, this study is on the gathering of complete and concrete floral data so that these expansion sites will become part of the protected area and encompassed in legal promulgations. Botanical fieldworks conducted from Oct to Dec 2017 were carried out in five study sites of the MHRWS expansion sites using $4020 \times 20 \mathrm{~m}$ sampling plot with a distance of $20 \mathrm{~m}$ between plots and opportunistic transect walk techniques. The study disclosed 228 taxa of plants, of these, 74 species were ferns and lycophytes, 6 species of gymnosperms, 30 species of herbs and vines and 118 species were trees and shrubs. There were three new records of ferns and lycophytes increasing the number of species to 155 . There are $13(5.7 \%)$ threatened species, $22(9.6 \%)$ and endemic species. Findings suggest that species in each site are unique and maybe attributed to the vegetation present, elevation variations of the different sampling sites and anthropogenic activities. The proposed expansion sites harbor diverse threatened and plants deserving protection and conservation efforts. Results of this study support the contention that the expansion sites, which are included in the municipal ordinances, be part of the official protected area.
\end{abstract}

KEYWORDS: biodiversity, Mt. Hamiguitan Range Wildlife Sanctuary expansion sites, Mindanao, Philippines, buffer zone

\section{INTRODUCTION}

The Philippines is a home of about 9,500 native vascular plant species, which is about $5 \%$ of the World's Flora (Pelser et al. 2011). It is considered as one of the world's eight biodiversity hottest hotspots (Myers et al. 2000).

Mt. Hamiguitan is home to 878 species of plants (Amoroso \& Aspiras 2011). Of these, 698 are angiosperms, 25 gymnosperms, 155 ferns and lycophytes which are at risk due to forest degradation and conversion of forested land to agriculture, shifting cultivation, and over-collection.

${ }^{*}$ Center for Biodiversity Research and Extension in Mindanao (CEBREM) Central, Mindanao University, Musuan Bukidnon, Philippines

*Corresponding email: victorbamoroso@gmail.com

Date Submitted: 21 March 2018

Date Accepted: 15 January 2019
Mt. Hamiguitan Range Wildlife Sanctuary (MHRWS) is a protected area by virtue of R.A. 9303 of July 30, 2004 following the objective of NIPAS Act of 1992 or R.A. 7586 (UNESCO 2014). The mountain totals an area of 169.23 $\mathrm{km}^{2}$ with the highest elevation of 1,637 $\mathrm{m}$ a.s.I. (UNESCO 2014) and characterized by a variety of vegetation types including a unique mossy pygmy forest (Amoroso et al. 2009). It is the only mountain peak in Mindanao with a pygmy forest inhabited by unique flora and fauna thus considered as hottest of the "hotspots" (Ong et al. 2002). The mountain is a UNESCO World Heritage Site, ASEAN Heritage Park and also a Mindanao Long Term Ecological Research (LTER) site.

In 2016, municipal ordinances to expand the protected area of the MHRWS were issued with the aim of protecting and preserving the remaining biodiversity of the buffer zones and to fortify the core zone. Thus, this study provides data on floral assemblage in the about $2.99 \mathrm{~km}^{2}$ expansion sites. The data will be an input to the Protected Area Suitability Assessment (PASA) as required under NIPAS law (R.A. 
7586) for appropriate legal promulgations.

\section{MATERIALS AND METHODS}

\section{Selection of Study sites and Obtaining of Permits.}

The study sites were identified and selected by the DENR Region XI personnel and researchers from Central Mindanao University. The study sites were limited to forest communities within the buffer zone and proposed expansion sites of the MHRWS, which were targeted for inclusion to the protected area. The five study sites, were distributed in the municipalities of San Isidro, Governor Generoso and Mati City (Fig. 1) which were botanically explored after a Wildlife Gratuitous Permit was secured from the Department of Environment and Natural Resources (DENR). The Prior Informed Consent (PIC) from the community was obtained by presenting the overview of the research specifically its objectives. The sites consisted of lowland mixed dipterocarp forest 118-622 $\mathrm{m}$ above sea level, slope to rolling plane (10$\left.45^{\circ}\right)$, ultramafic, emergent trees were Shorea polysperma (Blanco) Merr., Ochrosia spp. and Gymnostoma rumphianum (Miq.) L.A.S. Johnson reaching heights up to $32 \mathrm{~m}$ high and up to $65 \mathrm{~cm}$ dbh.

\section{Establishment of Sampling Plots and Field Sampling.}

Botanical fieldworks were carried out in the proposed MHRWS expansion sites from October to December 2017. A total of $4020 \times 20 \mathrm{~m}$ sampling plots with a distance of $20 \mathrm{~m}$ between each plot were established in the five sampling sites with eight plots per site to compare the alpha and beta diversity. Each plot served as the area for the inventory, diversity studies and assessment. Transect walk was likewise employed in between sampling plots to enrich the data on species richness.

\section{Sampling, Processing, Identification and Data Treatment.}

Inventory and listing of the different species of trees with at least $10 \mathrm{~cm}$ diameter at breast height $(\mathrm{DBH})$, ferns, lycophytes and other flowering plants inside each $20 \times 20 \mathrm{~m}$ sampling plots were done. The $\mathrm{DBH}$ of trees were measured using a diameter tape and the height with the use of laser dendrometer. Representative specimens of vascular plants were collected using the wet method technique, pressed and mounted as herbarium vouchers following standard procedures.

Classification and identification employed the use of books, taxonomic keys, checklist, online database (i.e. Global Plants on JSTOR, Co's Digital Flora of the Philippines) and literature. Plant voucher specimens were deposited at the Central Mindanao University Herbarium (CMUH). Photographs and photomicrographs were taken from suitable, representative plant specimen's in-situ.

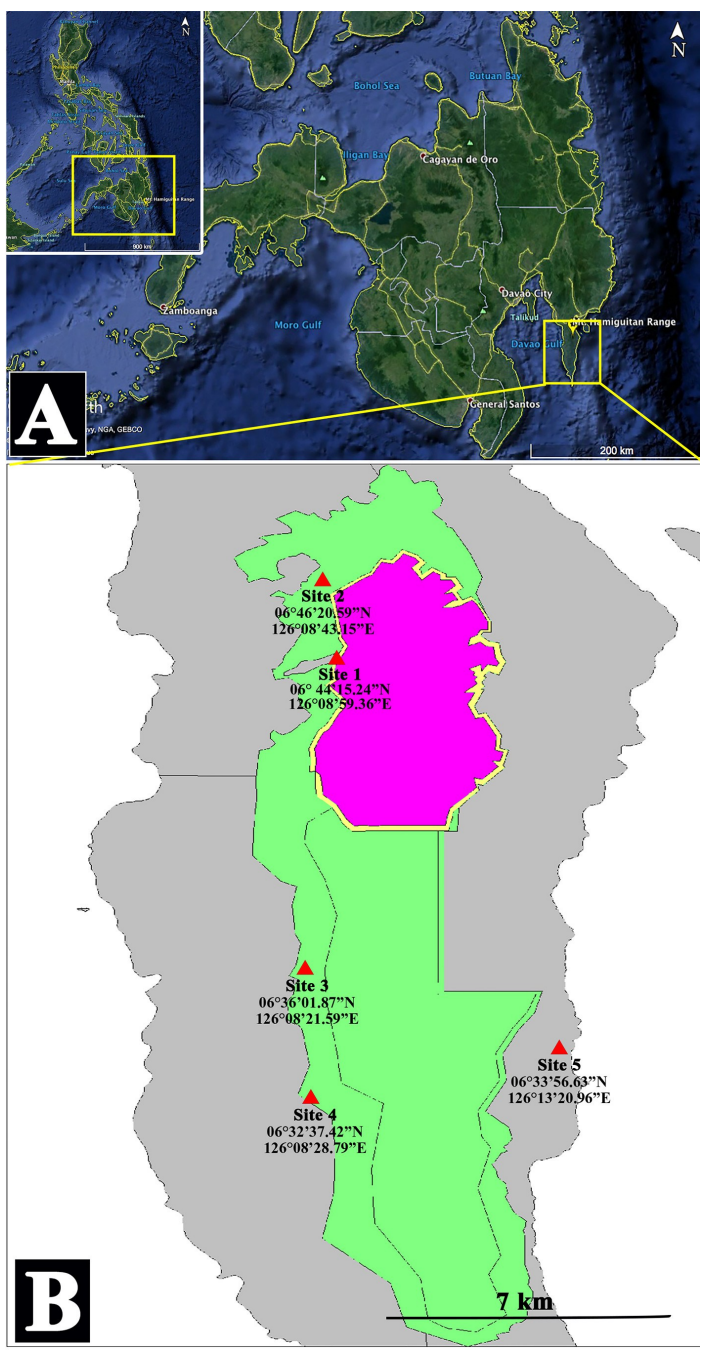

Figure 1. The study sites at Mt. Hamiguitan Range Wildlife Sanctuary expansion sites. A) Mindanao Map, (Philippines, inset), B) Study Sites. Site 1- Sitio Tumalite, Barangay La Union, San Isidro, Davao Oriental (622 masl); Site 2- Sitio Tibanga, Barangay Maputi, San Isidro, Davao Oriental (292 masl); Site 3Sitio Tagibo, Barangay Oregon, Governor Generoso, Davao Oriental (169 masl); Site 4- Sitio Tagaytay, Barangay Luzon, Governor Generoso, Davao Oriental (175 masl) Site 5- Sitio llib, Barangay Cabuaya, Mati City, Davao Oriental (118 masl)

The different parameters for measuring the magnitude of species diversity such as relative density, relative frequency, relative dominance, Shannon index of general diversity $(H)$ and Species Importance Value (SIV) were derived using the following formula: a) for trees $\mathrm{H}=-\mathrm{S}$ (dbh/Ndbh) log (dbh/ $\mathrm{Ndbh}$ ), where ndbh is the diameter at breast hight of individual tree species and $\mathrm{Ndbh}$ is the total diameter at breast height of all tree species ; b) for pteridophytes $\mathrm{H}=-\mathrm{S}$ [ni]/N log [ni]/N, where $\mathrm{N}$ is the total number of individuals in an area and c) SIV or ni = RD+RF+ Rdom, where RD = Relative Density, RF = Relative Frequency and Rdom = Relative Dominance. 


\section{RESULTS AND DISCUSSION}

\section{Species Composition}

Field inventory in the MHRWS expansion sites revealed 228 taxa of plants, which are distributed to 96 families and 171 genera (see Table 1). Of these, 74 species were ferns and lycophytes belonging to 24 families and 49 genera (see Table 4), 6 species of gymnosperms belonging to 3 families and 5 genera (see Table 2), 30 species were herbs and vines (flowering plants) placed in 17 families and 27 genera (see Table 3) and 118 species were trees and shrubs (seed plants) distributed among 52 families and 90 genera (see Table 2).

The floral species composition within the expansion sites is relatively high constituting about $26 \%$ of the total number of species found in entire MHRWS as reported by Amoroso and Aspiras (2011). The documented ferns and lycophytes comprise $49 \%$ of the total number in the entire range as reported by Amoroso et al. (2016). The new records of ferns and lycophytes in the Mt. Hamiguitan Range include Phlegmariurus phlegmaria (Lycopodiaceae), Pityrogramma calomelanos (Pteridaceae) and Drynaria sparsisora (Desv.) T. Moore (Polypodiaceae) increasing the species to 155 based on the most recent checklist (Amoroso et al. 2016).

The estimate of species richness of ferns and lycophytes of the entire range (155 spp.) closely resembles to the Karst Forest in Bohol Island with 169 spp. (Barcelona et al. 2006), Mt. Bali-it in Balbalasang-Balbalan National Park, and Northern Luzon with 167 ssp. (Barcelona 2003), Marilog Forest reserve in the area of Davao City with 165 spp. (Amoroso et al. 1996). The site harbors more species than Mt. Pinamantawan in Quezon of Bukidnon Province with 121 spp. (Sumagaysay 2012), Mt. Iraya and its vicinity in Batan Island, Batanes Province with 89 spp. (Barcelona 2003), Mt. Pangasugan on Leyte Island with 94 spp. (Belonias \& Banoc 1994), Pasonanca Natural Park in the region of Zamboanga City with 72 spp. (Andas 2015), and Mt. Makulot in Batangas Province, Southern Luzon with $40 \mathrm{spp}$. (Catapang et al. 2012). However, it is relatively lower than that of Mt. Kitanglad, Bukidnon Province with $439 \mathrm{spp}$. (Amoroso et al. 2011), Panay Island with 228 spp. (Barcelona 2004), Mt.
Burnay and its vicinity in Northern Luzon with 199 spp. (Iwatsuki \& Price 1977), and Mt. Malindang, Misamis Occidental Province with 280 spp. (Amoroso et al. 2006).

The families of plants with the highest number of species are Polypodiaceae (11 species), Pteridaceae (9), Selaginellaceae (5), Aspleniaceae, Davalliaceae, Lindsaeaceae, Tectariaceae and Thelypteridaceae with 4 species each, Rubiaceae (16 species), Orchidaceae (12 species), Myrtaceae (15), Moraceae (6), Apocynaceae (7), Fabaceae (5), and the rest of the families have at most 3 species. The common families of ferns are also widely distributed in Mindanao i.e. Mt. Apo (North Cotabato), Mt. Kitanglad (Bukidnon), Mt. Malindang (Misamis Occidental), Mt. Hamiguitan (Davao Oriental) and Mt. Musuan (Bukidnon) (Amoroso et al. 2009, 2015). The fern families with highest number of species in the expansion sites also contain the highest number of species in the Philippines (Salgado 1990).

\section{Diversity Index Values}

The average diversity index value of trees $\left(H^{\prime}=1.3\right)$ in the proposed expansion sites is comparatively lower compared to the $0.01 \mathrm{~km}^{2}$ permanent plot in Mt. Musuan, Bukidnon $\left(\mathrm{H}^{\prime}\right.$ =1.93), Mt. Malindang, Misamis Occidental $\left(H^{\prime}=1.68\right)$, Mt. Apo $\left(H^{\prime}=1.57\right)$, Mt. Hamiguitan $\left(H^{\prime}=1.57\right)$ and Mt. Kitanglad $\left(H^{\prime}=1.43\right)$ (see Table 5).

The diversity index value of pteridophytes in the proposed expansion sites was relatively lower with average diversity value of $H^{\prime}=0.6$, compared to the permanent plot of Mt. Malindang, Misamis Occidental $\left(H^{\prime}=1.41\right)$, Mt. Kitanglad, Bukidnon $\left(H^{\prime}=1.37\right)$, Mt. Apo $\left(H^{\prime}=1.26\right)$, Mt. Musuan, Bukidnon $\left(H^{\prime}=1.01\right)$ and Mt. Hamiguitan $\left(H^{\prime}=0.91\right)$ (Amoroso et al. 2015) (see Table 5).

\section{Species Importance Value (SIV)}

The species with high Species Importance Value (SIV) for ferns and lycophytes in all the study sites include: Taenitis blechnoides, Selaginella jagorii, Sphaeropteris polypoda, Schizaea dichotoma, Pteridium aquilinum, Tectaria laxa, Lindsaea gueriniana, Adiantum hosei, Pyrrosia lanceolata,

Table 1. Species composition of flora in MHRWS expansion sites.

\begin{tabular}{lccc}
\hline Plant Group & No. of Families & No. of Genera & No. of Species \\
\hline Ferns and Lycophytes & 24 & 49 & 74 \\
Gymnosperms & 3 & 5 & 6 \\
Herbs and Vines (flowering plants) & 17 & 27 & 30 \\
Trees and Shrubs (seed plants) & 52 & 90 & 118 \\
\hline Total & 96 & 171 & 228 \\
\hline
\end{tabular}


Philippine Journal of Systematic Biology | Amoroso,V.B. et al.: Floral diversity assessment of the MHRWS

Table 2. Checklist of trees and shrubs in the MHRWS expansion sites

\begin{tabular}{|c|c|c|c|c|}
\hline Collection No. & Family & Species & $\begin{array}{c}\text { Conservation } \\
\text { Status }\end{array}$ & $\begin{array}{c}\text { Ecological } \\
\text { Status }\end{array}$ \\
\hline & Angiosperms & & & \\
\hline & Acanthaceae & Justicia sp. & - & - \\
\hline & Actinidiaceae & Saurauia sp. & - & - \\
\hline & & Saurauia avellana Elmer & - & - \\
\hline \multirow[t]{2}{*}{ VBA 7221} & Anacardiaceae & Buchanania arborescens (Blume) & & \\
\hline & & Blume & - & - \\
\hline \multirow[t]{5}{*}{ VBA 7192} & & Semecarpus sp. & - & - \\
\hline & Annonaceae & Huberantha rumphii (Blume ex & & \\
\hline & & Hensch.) Chaowasku & - & - \\
\hline & Apocynaceae & Alstonia scholaris (L.) R.Br. & - & - \\
\hline & & Melodinus philippinensis A. Dc. & - & PE \\
\hline VBA 7184 & & Ochrosia apoensis Elmer & - & - \\
\hline VBA 7390 & & Ochrosia glomerata (Blume) F. Muell. & - & - \\
\hline \multirow[t]{7}{*}{ VBA 7202} & & Tabernaemontana pandacaqui Lam. & - & - \\
\hline & Araliaceae & Osmoxylon luzoniense (Merr.) & & \\
\hline & & Philipson & - & - \\
\hline & & Polyscias aherniana (Merr.) Lowry \& & & \\
\hline & & G.M.Plunkett & - & - \\
\hline & Arecaceae & Pinanga sp. & - & - \\
\hline & Bignoniaceae & Radermacherasp. & - & - \\
\hline \multirow{4}{*}{$\begin{array}{l}\text { VBA } 7400 \\
\text { VBA } 7220\end{array}$} & Burseraceae & Canarium asperum Benth. & - & - \\
\hline & & & & \\
\hline & Calophyllaceae & Calophyllum mindanaense EImer & - & - \\
\hline & Cannabaceae & Celtis sp. & - & - \\
\hline \multirow[t]{5}{*}{ VBA 7215} & Casuarinaceae & Gymnostoma rumphianum (Miq.) & & \\
\hline & & L.A.S. Johnson & - & - \\
\hline & Clusiaceae & Garcinia sp. & - & - \\
\hline & & Mammea ultramafica P. F. Stevens & - & - \\
\hline & Cunoniaceae & Weinmannia sp. & - & - \\
\hline \multirow[t]{2}{*}{ VBA 7213} & Dilleniaceae & Dillenia philippinensis Rolfe & - & $\mathrm{PE}$ \\
\hline & Dipentodontaceae & Perrottetia sp. & - & - \\
\hline \multirow[t]{2}{*}{ VBA 7177} & Dipterocarpaceae & Shorea astylosa Foxw. & $\mathrm{CR}$ & PE \\
\hline & & Shorea polysperma Merr. & VU & PE \\
\hline
\end{tabular}


Philippine Journal of Systematic Biology | Amoroso,V.B. et al.: Floral diversity assessment of the MHRWS

Table 2 cont. Checklist of trees and shrubs in the MHRWS expansion sites

\begin{tabular}{|c|c|c|c|c|}
\hline Collection No. & Family & Species & $\begin{array}{c}\text { Conservation } \\
\text { Status }\end{array}$ & $\begin{array}{c}\text { Ecological } \\
\text { Status }\end{array}$ \\
\hline & Ebenaceae & Diospyros sp. & - & - \\
\hline VBA 7380 & Elaeocarpaceae & Elaeocarpus sp. & - & - \\
\hline \multirow[t]{4}{*}{ VBA 7371} & Ericaceae & Vaccinium agusanense Elmer & - & ME \\
\hline & Euphorbiaceae & Macaranga gigantifolia Merr. & - & - \\
\hline & & Macaranga sp. & - & - \\
\hline & & Sauropus villosus (Blanco) Merr. & - & - \\
\hline \multirow[t]{4}{*}{ VBA 7385} & & Sauropus sp. & - & - \\
\hline & & & & \\
\hline & Fabaceae & Archidendron ellipticum (Blanco) & - & \\
\hline & & I.C. Nielsen & & \\
\hline \multirow[t]{10}{*}{ VBA 7397} & & Callerya scandens (Elmer) Schot & - & - \\
\hline & & Cynometrasp. & - & - \\
\hline & & Ormosia sp. & - & - \\
\hline & Fagaceae & Lithocarpus sulitii Soepadmo & - & PE \\
\hline & & Lithocarpus woodii (Hance) A.Camus & - & - \\
\hline & & Lithocarpus sp. & - & - \\
\hline & & Castanopsis evansii Elmer & - & PE \\
\hline & Gentianaceae & Fagraea philippinensis K.M.Wong & & \\
\hline & & \& Sugau & - & - \\
\hline & Hypericaceae & Cratoxylum sp. & - & - \\
\hline \multirow[t]{5}{*}{ VBA 7405} & Lamiaceae & Callicarpa flavida EImer & - & - \\
\hline & & Teijsmanniodendron ahernianum & & \\
\hline & & (Merr.) Bakh. & - & - \\
\hline & Lauraceae & Actinodaphne akoensis var hayatae & & \\
\hline & & (Kaneh.) J.C. Liao & - & - \\
\hline \multirow[t]{7}{*}{ VBA 7126} & & Cinnamomum mercadoi Vidal & OTS & - \\
\hline & & Cinnamomum sp. & - & - \\
\hline & & Litsea sp. & - & - \\
\hline & Lecythidaceae & Barringtonia racemosa (L.) Spreng & - & - \\
\hline & & Barringtonia sp. & - & - \\
\hline & Melastomataceae & Astronia sp. & - & - \\
\hline & & Medinilla multiflora Merr. & - & - \\
\hline VBA 7208 & & Melastoma malabathricum $\mathrm{L}$. & - & - \\
\hline
\end{tabular}


Philippine Journal of Systematic Biology | Amoroso,V.B. et al.: Floral diversity assessment of the MHRWS

Table 2 cont. Checklist of trees and shrubs in the MHRWS expansion sites

\begin{tabular}{|c|c|c|c|c|}
\hline Collection No. & Family & Species & $\begin{array}{c}\text { Conservation } \\
\text { Status }\end{array}$ & $\begin{array}{l}\text { Ecological } \\
\text { Status }\end{array}$ \\
\hline & Meliaceae & Aglaia luzoniensis Merr. \& Rolfe & - & - \\
\hline & Moraceae & Artocarpus multifidus F.M. Jarrett & - & - \\
\hline & & Streblus elongatus (Miq.) Corner & - & - \\
\hline \multirow[t]{4}{*}{ VBA 7257} & & Ficus botryocarpa Miq. & - & - \\
\hline & & Ficus pseudopalma Blanco & - & - \\
\hline & & Ficus elmeri var. subintegra Merr. & - & - \\
\hline & & Ficus sp. & - & - \\
\hline VBA 7267 & Myristicaceae & Horsfieldia sp. & - & - \\
\hline \multirow[t]{3}{*}{ VBA 7403} & Myrsinaceae & Myrsine glandulosa (Elmer) Pipoly & - & - \\
\hline & Myrtaceae & Leptospermum javanicum Blume & - & - \\
\hline & & Rhodomyrtus surigaoensis Elmer & - & - \\
\hline \multirow[t]{6}{*}{ VBA 7204} & & Syzygium antisepticum (Blume) Merr. & & \\
\hline & & \& L.M.Perry & - & ME \\
\hline & & Syzygium incarnatum (Elmer) Merr. \& & & \\
\hline & & L.M. Perry & - & - \\
\hline & & Syzygium rubrovenium (C. B. Rob.) & & \\
\hline & & Merr. & - & - \\
\hline VBA 7391 & & Syzygium sp. 1 & - & - \\
\hline VBA 7370 & & Syzygium sp. 2 & - & - \\
\hline VBA 7373 & & Syzygium sp. 3 & - & - \\
\hline VBA 7386 & & Syzygium sp. 4 & - & - \\
\hline \multirow[t]{12}{*}{ VBA 7219} & & Syzygium sp. 5 & - & - \\
\hline & & Syzygium sp. 6 & - & - \\
\hline & & Syzygium sp. 7 & - & - \\
\hline & & Syzygium sp. 8 & - & - \\
\hline & & Tristaniopsis decorticata (Merr.) Peter & & \\
\hline & & G.Wilson \& J.T. Waterh. & VU & - \\
\hline & Pandanaceae & Benstonea copelandii (Merr.) Callm. & & \\
\hline & & \& Buerki & - & - \\
\hline & & Pandanus clementis Merr. & - & - \\
\hline & & Sararanga sinuosa Hemsl. & - & - \\
\hline & Pentaphylacaceae & Euryasp. & - & - \\
\hline & & Ternstroemia philippinensis Merr. & - & - \\
\hline
\end{tabular}


Philippine Journal of Systematic Biology | Amoroso,V.B. et al.: Floral diversity assessment of the MHRWS

Table 2 cont. Checklist of trees and shrubs in the MHRWS expansion sites

\begin{tabular}{|c|c|c|c|c|}
\hline Collection No. & Family & Species & $\begin{array}{l}\text { Conservation } \\
\text { Status }\end{array}$ & $\begin{array}{c}\text { Ecological } \\
\text { Status }\end{array}$ \\
\hline VBA 7216 & Phyllanthaceae & Antidesma sp. & - & - \\
\hline \multirow[t]{3}{*}{ VBA 7266} & & Breynia cernua Müll.Arg. & - & - \\
\hline & Pittosporaceae & Pittosporum euphlebium Merr. & - & - \\
\hline & Polygalaceae & Polygala venenosa Juss. ex Poir. & - & - \\
\hline VBA 7412 & Primulaceae & Ardisia sp. & - & - \\
\hline VBA 7203 & Proteaceae & Helicia paucinervia Merr. & - & - \\
\hline \multirow[t]{4}{*}{ VBA 7347} & Rhamnaceae & Alphitonia excelsa (Fenzl) Benth. var. & - & - \\
\hline & & excelsa (Fenzl) Benth. & & \\
\hline & Rosaceae & Prunus sp. & - & - \\
\hline & Rubiaceae & Greeniopsis multiflora (Elmer) Merr. & - & - \\
\hline \multirow[t]{3}{*}{ VBA 7375} & & Greeniopsis sp. & - & - \\
\hline & & Ixora philippinensis Merr. & - & PE \\
\hline & & Ixorasp. & - & - \\
\hline \multirow[t]{6}{*}{ VBA 7348} & & Morinda coriacea Merr. & - & - \\
\hline & & Morinda citrifolia L & - & - \\
\hline & & Mussaenda philippica A.Rich. & - & - \\
\hline & & var. philippica & & \\
\hline & & Nauclea wenzelii Merr. & - & - \\
\hline & & Nauclea sp. & - & - \\
\hline \multirow[t]{2}{*}{ VBA 7205} & & Psychotria mariguidonensis Sohmer & - & - \\
\hline & & \& A.P.Davis & & \\
\hline VBA 7406 & & Psychotria sp. & - & - \\
\hline VBA 7380 & & Timonius trichophorus Merr. & - & - \\
\hline \multirow[t]{3}{*}{ VBA 7402} & & Timonius sp. & - & - \\
\hline & & Wendlandia nervosa Merr. & - & - \\
\hline & Rutaceae & Adenandra sp. & - & - \\
\hline \multirow[t]{3}{*}{ VBA 7381} & & Lunasia amara Blanco & - & - \\
\hline & & Melicope sp. & - & - \\
\hline & & Zanthoxylum diabolicum Elmer & - & - \\
\hline VBA 7376 & & Acronychiasp. & - & - \\
\hline VBA 7180 & Sapindaceae & Ganophyllum falcatum Blume & - & - \\
\hline VBA 7199 & & Guioa discolor Radlk. & - & - \\
\hline VBA 7384 & Sapotaceae & Palaquium sp. & - & - \\
\hline
\end{tabular}


Philippine Journal of Systematic Biology | Amoroso,V.B. et al.: Floral diversity assessment of the MHRWS

Table 2 cont. Checklist of trees and shrubs in the MHRWS expansion sites

\begin{tabular}{|c|c|c|c|c|}
\hline Collection No. & Family & Species & $\begin{array}{l}\text { Conservation } \\
\text { Status }\end{array}$ & $\begin{array}{c}\text { Ecologica } \\
\text { Status }\end{array}$ \\
\hline & Staphyleaceae & Turpinia ovalifolia Elmer & - & - \\
\hline \multirow[t]{2}{*}{ VBA 7392} & Stemonuraceae & Gomphandra cf. apoensis & - & - \\
\hline & Sterculiaceae & Sterculia sp. & - & - \\
\hline VBA 7206 & & Commersonia bartramia (L.) Merr. & - & - \\
\hline \multirow[t]{8}{*}{ VBA 7200} & Urticaceae & Leucosyke elmeri Unruh & - & - \\
\hline & Vitaceae & Leea guineensis G. Don & - & - \\
\hline & Gymnosperms & & & \\
\hline & Araucariaceae & Agathis philippinensis Warb. & - & - \\
\hline & Gnetaceae & Gnetum gnemon L. & - & - \\
\hline & & Gnetum latifolium Blume & - & - \\
\hline & Podocarpaceae & Falcatifolium gruezoi de Laub. & - & - \\
\hline & & Nageia wallichiana Kuntze & - & - \\
\hline VBA 7399 & & Podocarpus philippinensis Foxw. & - & - \\
\hline
\end{tabular}

No available reproductive organs for taxa identified only to the genus level

CR- Critically Endangered, VU- Vulnerable, OTS- Other Threatened Species, ME- Mindanao Endemic, PE- Philippine Endemic

Table 3. Checklist of other flowering plants (herbs \& vines) in the MHRWS expansion sites

\begin{tabular}{llcc}
\hline \multicolumn{1}{c}{ Family } & \multicolumn{1}{c}{ Species } & $\begin{array}{c}\text { Conservation } \\
\text { Status }\end{array}$ & $\begin{array}{c}\text { Ecological } \\
\text { Status }\end{array}$ \\
\hline Apocynaceae & Dischidia major (Vahl) Merr. & - & - \\
Hraceae & Hoya sp. & - & - \\
& Pothos sp. 1 & - & - \\
Arecaceae & Pothos sp. 2 & - & - \\
Dilleniaceae & Calamus sp. & - & - \\
Fabaceae & Tetracera scandens (L.) Merr. & - & - \\
Flagellariaceae & Bauhinia pauciflora Merr. & - & - \\
Goodeniaceae & Flagellaria indica Linn. & - & - \\
Loranthaceae & Scaevola pedunculata Merr. & - & - \\
Melastomataceae & Amyema celebica Danser & - & - \\
Nepenthaceae & Sarcopyramis sp. & Nepenthes mindanaoensis Kurata & VU \\
& Nepenthes alfredoi Amoroso \& Lagunday & CR & PE \\
\hline
\end{tabular}


Philippine Journal of Systematic Biology | Amoroso,V.B. et al.: Floral diversity assessment of the MHRWS

Table 3 cont. Checklist of other flowering plants (herbs \& vines) in the MHRWS expansion sites

\begin{tabular}{|c|c|c|c|}
\hline Family & Species & $\begin{array}{c}\text { Conservation } \\
\text { Status }\end{array}$ & $\begin{array}{c}\text { Ecological } \\
\text { Status }\end{array}$ \\
\hline \multirow[t]{11}{*}{ Orchidaceae } & Bulbophyllum brevibrachiatum J.J.Sm. & - & PE \\
\hline & Cadetia microphyton (L.O. Williams) & & \\
\hline & Christenson & - & PE \\
\hline & Crepidium sp. & - & - \\
\hline & Liparis parviflora (Blume) Lindl. & - & - \\
\hline & Paphiopedilum ciliolare (Rchb.f.) Stein & CR & - \\
\hline & Podochilus intricatus Ames & - & - \\
\hline & Pteroceras sp. & - & - \\
\hline & Thecostele alata C.S.P.Parish \& Rchb.f. & - & - \\
\hline & Trichoglottis geminata J.J.Sm. & - & PE \\
\hline & Trichoglottis latisepala Ames & - & ME \\
\hline Pandanaceae & Freycinetia cumingiana Gaudich. & - & PE \\
\hline Pentaphragmataceae & Pentaphragma grandiflorum Kurz & - & - \\
\hline Piperaceae & Piper lessertianum C. DC. & - & - \\
\hline \multirow[t]{2}{*}{ Rubiaceae } & Hydnophytum formicarum Jack & - & - \\
\hline & Myrmecodia tuberosa Jack & - & - \\
\hline \multirow[t]{2}{*}{ Smilacaceae } & Smilax bracteata var. heterophylla & & \\
\hline & Merr. \& Quisumb. & - & - \\
\hline Zingiberaceae & Alpinia musifolia Ridl. & - & - \\
\hline
\end{tabular}

No available reproductive organs for taxa identified only to the genus level CR- Critically Endangered, VU- Vulnerable, OTS- Other Threatened Species, ME- Mindanao Endemic, PE- Philippine Endemic 
Philippine Journal of Systematic Biology | Amoroso,V.B. et al.: Floral diversity assessment of the MHRWS

Table 4. Checklist of ferns and lycophytes in the MHRWS expansion sites

\begin{tabular}{|c|c|c|c|c|}
\hline Collection No. & Family & Species & $\begin{array}{c}\text { Conservation } \\
\text { Status }\end{array}$ & $\begin{array}{c}\text { Ecological } \\
\text { Status }\end{array}$ \\
\hline \multirow[t]{2}{*}{ VBA 7398} & Lycopodiaceae & Phlegmariurus phlegmaria & & \\
\hline & & (L.) Holub & EN & - \\
\hline VBA 7324 & Selaginellaceae & Selaginella alligans Hieron. & - & - \\
\hline VBA 7269 & & S. cupressina (Willd.) Spring & - & - \\
\hline VBA 7155 & & S. involvens (Sw.) Spring & - & - \\
\hline VBA 7102 & & S. jagorii Warb. & - & PE \\
\hline \multirow[t]{2}{*}{ VBA 7036} & & Selaginella sp.1 & - & - \\
\hline & Aspleniaceae & Asplenium excisum C. Presl & - & - \\
\hline VBA 7161 & & A. nigrescens Hook. f. & - & - \\
\hline \multirow[t]{10}{*}{ VBA 7251} & & A. polyodon G. Forst. & - & - \\
\hline & & A. tenerum G. Forst. & - & - \\
\hline & Athyriaceae & Athyrium puncticaule & & \\
\hline & & (Blume) T. Moore & - & - \\
\hline & & Diplazium sp. & - & - \\
\hline & Blechnaceae & Blechnum egregium Copel. & - & - \\
\hline & Cyatheaceae & Sphaeropteris elmeri & & \\
\hline & & R.M.Tryon & VU & $\mathrm{PE}$ \\
\hline & & S. glauca (Blume) R.M. Tryon & EN & - \\
\hline & & S. polypoda R.M.Tryon & - & - \\
\hline VBA 7243 & Davalliaceae & Davallia heterophylla Sm. & - & - \\
\hline \multirow[t]{5}{*}{ VBA 7025} & & D. solida (G. Forster) Swartz & - & - \\
\hline & & Davallodes hirsutum & & \\
\hline & & (J.Sm.) Copel. & - & - \\
\hline & & Humata repens (L. f.) J. Small & & \\
\hline & & ex Diels & - & - \\
\hline \multirow[t]{3}{*}{ VBA 7156} & Dennstaedtiaceae & Orthiopteris campylura & & \\
\hline & & (Kunze) Copel. & - & - \\
\hline & & Pteridium aquilinum (L.) Kuhn & - & - \\
\hline \multirow[t]{4}{*}{ VBA 7286} & Dicksoniaceae & Calochlaena javanica & & \\
\hline & & (BI.) G.B.Nair & - & - \\
\hline & Dryopteridaceae & Ctenitis sp. & - & - \\
\hline & & Dryopteris sp. & - & - \\
\hline VBA 7101 & & Lomagramma pteroides J. Sm. & - & - \\
\hline
\end{tabular}


Philippine Journal of Systematic Biology | Amoroso,V.B. et al.: Floral diversity assessment of the MHRWS

Table 4 cont. Checklist of ferns and lycophytes in the MHRWS expansion sites

\begin{tabular}{|c|c|c|c|c|}
\hline Collection No. & Family & Species & $\begin{array}{l}\text { Conservation } \\
\text { Status }\end{array}$ & $\begin{array}{c}\text { Ecological } \\
\text { Status }\end{array}$ \\
\hline & Gleicheniaceae & Dicranopteris curranii Copel. & - & - \\
\hline & & Dicranopteris linearis (Burm.f.) & & \\
\hline & & Underw. & - & - \\
\hline & Hymenophyllaceae & Diplopterygium longissimum & & \\
\hline & & (Blume) Nakai & - & - \\
\hline \multirow[t]{2}{*}{ VBA 7104} & & Cephalomanes atrovirens & & \\
\hline & & C. Presl & - & - \\
\hline \multirow[t]{2}{*}{ VBA 7182} & Lindsaeaceae & Lindsaea gueriniana (Gaudich.) & & \\
\hline & & Desv. & - & - \\
\hline \multirow[t]{4}{*}{ VBA 7108} & & L. hamiguitanensis Karger & & \\
\hline & & \& V.B.Amoroso & OTS & ME \\
\hline & & Odontosoria retusa (Cav.) & & \\
\hline & & J. Sm. & - & - \\
\hline \multirow[t]{2}{*}{ VBA 7024} & & Tapeinidium Iuzonicum (Hook.) & & \\
\hline & & K.U. Kramer & - & - \\
\hline \multirow[t]{6}{*}{ VBA 7275} & Lomariopsidaceae & Nephrolepis biserrata & & \\
\hline & & (Sw.) Schot & - & - \\
\hline & & N. cordifolia (L.) PresI & - & - \\
\hline & & N. hirsutula (G. Forst.) C. Presl & - & - \\
\hline & Lygodiaceae & Lygodium circinnatum & & \\
\hline & & (Burm. fil.) Sw. & - & - \\
\hline \multirow[t]{9}{*}{ VBA 7228} & & L. flexuosum (L.) Sw. & - & - \\
\hline & Marattiaceae & Angiopteris evecta (G.Forst.) & & \\
\hline & & Hoffm. & - & - \\
\hline & Ophioglossaceae & Ophioderma reticulatum $\mathrm{L}$. & - & - \\
\hline & Osmundaceae & Osmunda banksiifolia & & \\
\hline & & (Presl) Kuhn & - & - \\
\hline & Polypodiaceae & Drynaria descensa Copel. & - & - \\
\hline & & D. quercifolia (L.) J.Sm & - & - \\
\hline & & D. sparsisora (Desv.) T. Moore & - & - \\
\hline \multirow[t]{2}{*}{ VBA 7142} & & Lecanopteris sinuosa & & \\
\hline & & (Wall. ex Hook.) Copel. & VU & - \\
\hline
\end{tabular}


Philippine Journal of Systematic Biology | Amoroso,V.B. et al.: Floral diversity assessment of the MHRWS

Table 4 cont. Checklist of ferns and lycophytes in the MHRWS expansion sites

\begin{tabular}{|c|c|c|c|c|}
\hline Collection No. & Family & Species & $\begin{array}{l}\text { Conservation } \\
\text { Status }\end{array}$ & $\begin{array}{c}\text { Ecological } \\
\text { Status }\end{array}$ \\
\hline \multirow[t]{2}{*}{ VBA 7150} & & Lepisorus longifolius (Blume) & & \\
\hline & & Holttum & - & - \\
\hline VBA 7250 & & Microsorum scolopendria & & \\
\hline \multirow[t]{4}{*}{ VBA 7299} & & (Burm f.) Copel. & - & - \\
\hline & & Platycerium coronarium & $\mathrm{CR}$ & - \\
\hline & & (J. Koenig ex O. F. Müll.) & & \\
\hline & & Desv. & & \\
\hline \multirow[t]{2}{*}{ VBA 7201} & & Pyrrosia adnascens (Swartz) & & \\
\hline & & Ching. & - & - \\
\hline VBA 7374 & & P. lanceolata (L.) Farw. & - & - \\
\hline \multirow[t]{2}{*}{ VBA 7332} & & P. samarensis (C. Presl) Ching & - & PE \\
\hline & & Selliguea taeniata (Sw.) Parris & - & - \\
\hline VBA 7388 & Psilotaceae & Psilotum nudum (L.) Griseb. & VU & - \\
\hline \multirow[t]{2}{*}{ VBA 7322} & Pteridaceae & Adiantum hosei Baker & - & ME \\
\hline & & Antrophyum sp. & - & - \\
\hline \multirow[t]{4}{*}{ VBA 7302} & & Haplopteris ensiformis & & \\
\hline & & (Sw.) E.H. Crane & - & - \\
\hline & & Pityrogramma calomelanos & & \\
\hline & & (L.) Link & - & - \\
\hline VBA 7331 & & Pteris oppositipinnata Fée & - & $\mathrm{PE}$ \\
\hline \multicolumn{5}{|l|}{ VBA 7338} \\
\hline \multicolumn{5}{|l|}{ VBA 7252} \\
\hline VBA 7288 & & Pteris sp. & - & - \\
\hline \multirow[t]{3}{*}{ VBA 7367} & & Pteris vittata $\mathrm{L}$. & - & - \\
\hline & & Syngramma alismifolia & & \\
\hline & & (C. Presl) J. Sm. & - & - \\
\hline \multirow[t]{4}{*}{ VBA 7181} & & Taenitis blechnoides & & \\
\hline & & (Willd.) Sw. & - & - \\
\hline & Schizaeaceae & Schizaea dichotoma (L.) Sm. & - & - \\
\hline & & S. digitata (L.) Sw. & - & - \\
\hline \multirow[t]{3}{*}{ VBA 7106} & & S. inopinata Selling & - & - \\
\hline & Tectariaceae & Pleocnemia irregularis & & \\
\hline & & (C. Presl) Holttum & - & - \\
\hline
\end{tabular}


Philippine Journal of Systematic Biology | Amoroso,V.B. et al.: Floral diversity assessment of the MHRWS

Table 4 cont. Checklist of ferns and lycophytes in the MHRWS expansion sites

\begin{tabular}{|c|c|c|c|c|}
\hline Collection No. & Family & Species & $\begin{array}{l}\text { Conservation } \\
\text { Status }\end{array}$ & $\begin{array}{c}\text { Ecological } \\
\text { Status }\end{array}$ \\
\hline VBA 7264 & & Tectaria crenata Cav. & - & - \\
\hline \multirow[t]{2}{*}{ VBA 7162} & & T. polymorpha (Wall. ex Hook.) & & \\
\hline & & Copel. & - & - \\
\hline VBA 7327 & & T. laxa (Copel.) M.G.Price & - & PE \\
\hline \multirow[t]{2}{*}{ VBA 7287} & Thelypteridaceae & Christella dentata (Forsk.) & - & - \\
\hline & & Brownsey \& Jermy & & \\
\hline \multirow[t]{5}{*}{ VBA 7355} & & Macrothelypteris polypodioides & - & - \\
\hline & & (Hook.) Holttum & & \\
\hline & & Pronephrium nitidum Holttum & - & - \\
\hline & & Sphaerostephanos unitus & - & - \\
\hline & & (L.) Holttum & & \\
\hline
\end{tabular}

Table 5. Species richness and diversity values of trees and pteridophytes in MHRWS expansion sites

\begin{tabular}{|c|c|c|c|c|c|c|}
\hline \multirow[b]{2}{*}{ Site } & \multirow[b]{2}{*}{ Coordinates } & \multirow{2}{*}{$\begin{array}{l}\text { Elevation } \\
\text { (masl) }\end{array}$} & \multicolumn{2}{|c|}{ Trees } & \multicolumn{2}{|c|}{ Ferns \& Lycophytes } \\
\hline & & & $\begin{array}{c}\text { Species } \\
\text { Richness }\end{array}$ & $\begin{array}{l}\text { Diversity } \\
\text { Index (H) }\end{array}$ & $\begin{array}{l}\text { Species } \\
\text { Richness }\end{array}$ & $\begin{array}{l}\text { Diversity } \\
\text { Index }(H)\end{array}$ \\
\hline Site 1 & $\begin{array}{l}06^{\circ} 44^{\prime} 15.24 " \mathrm{~N} \\
126^{\circ} 08^{\prime} 59.36^{\prime \prime} \mathrm{E}\end{array}$ & 622 & 67 & 1.35 & 50 & 0.93 \\
\hline Site 2 & $\begin{array}{l}06^{\circ} 46^{\prime} 20.59^{\prime \prime} \mathrm{N} \\
126^{\circ} 08^{\prime} 43.15^{\prime \prime} \mathrm{E}\end{array}$ & 292 & 65 & 1.24 & 11 & 0.55 \\
\hline Site 3 & $\begin{array}{l}06^{\circ} 36^{\prime} 01.87^{\prime \prime N} \\
126^{\circ} 08^{\prime} 21.59^{\prime \prime} \mathrm{E}\end{array}$ & 169 & 70 & 1.38 & 33 & 0.71 \\
\hline Site 4 & $\begin{array}{l}06^{\circ} 32^{\prime} 37.422^{\prime \prime} \mathrm{N} \\
126^{\circ} 08^{\prime} 28.79^{\prime \prime} \mathrm{E}\end{array}$ & 175 & 50 & 1.10 & 19 & 0.07 \\
\hline Site 5 & $\begin{array}{l}06^{\circ} 33^{\prime} 56.63^{\prime \prime} \mathrm{N} \\
126^{\circ} 13^{\prime} 20.96^{\prime \prime} \mathrm{E}\end{array}$ & 118 & 45 & 1.21 & 14 & 0.73 \\
\hline Average & & & 59.4 & 1.3 & 25.4 & 0.6 \\
\hline
\end{tabular}


Table 6. Species with high Species Importance Value (SIV) in MHRWS expansion sites

\begin{tabular}{|c|c|}
\hline Species & SIV \\
\hline \multicolumn{2}{|l|}{ Site 1} \\
\hline Taenitis blechnoides (Willd.) Sw. & 77.32 \\
\hline Selaginella jagorii Warb. & 56.75 \\
\hline Sphaeropteris polypoda R.M.Tryon & 55.69 \\
\hline Macaranga sp. & 24.21 \\
\hline Teijsmanniodendron ahernianum (Merr.) Bakh. & 19.31 \\
\hline Lithocarpus woodii (Hance) A. Camus & 18.56 \\
\hline \multicolumn{2}{|l|}{ Site 2} \\
\hline Taenitis blechnoides (Willd.) Sw. & 160.62 \\
\hline Schizaea dichotoma (L.) Sm. & 47.60 \\
\hline Ochrosia glomerata Valeton & 30.36 \\
\hline Ochrosia apoensis Elmer & 23.66 \\
\hline Artocarpus multifidus F.M. Jarrett & 22.33 \\
\hline \multicolumn{2}{|l|}{ Site 3} \\
\hline Taenitis blechnoides (Willd.) Sw. & 100.79 \\
\hline Schizaea dichotoma (L.) Sm. & 89.51 \\
\hline Tectaria laxa (Copel.) M.G. Price & 47.93 \\
\hline Sloetia elongata Koord. & 26.33 \\
\hline Buchanania arborescens (Blume) Blume & 18.66 \\
\hline Greeniopsis multiflora (Elmer) Merr. & 17.98 \\
\hline \multicolumn{2}{|l|}{ Site 4} \\
\hline Lindsaea gueriniana (Gaudich.) Desv. & 120.18 \\
\hline Taenitis blechnoides (Willd.) Sw. & 95.15 \\
\hline Ochrosia glomerata Valeton & 24.00 \\
\hline Shorea astylosa Foxw. & 22.40 \\
\hline Gymnostoma rumphianum (Miq.) L.A.S. Johnson & 22.09 \\
\hline Teijsmanniodendron ahernianum (Merr.) Bakh. & 22.07 \\
\hline Palaquium sp. & 22.05 \\
\hline \multicolumn{2}{|l|}{ Site 5} \\
\hline Pyrrosia lanceolata (L.) Farw. & 88.63 \\
\hline Nephrolepis hirsutula (G. Forst.) C. Presl & 57.76 \\
\hline Lygodium circinnatum (Burm. fil.) Sw. & 57.76 \\
\hline Dicranopteris linearis (Burm.f.) Underw. & 54.27 \\
\hline Syzygium sp. & 37.56 \\
\hline Shorea astylosa Foxw. & 35.37 \\
\hline Ochrosia glomerata Valeton & 24.96 \\
\hline
\end{tabular}


Nephrolepis hirsutula and Lygodium circinnatum. It was found out that $T$. blechnoides is widely distributed in almost all the sites except in site 5 . This species ranks $1^{\text {st }}$ or $2^{\text {nd }}$ most dominant species of ferns within the Mt. Hamiguitan expansion site (see Table 6).

For the trees, Macaranga sp., Teijsmanniodendron ahernianum, Lithocarpus woodii, Ochrosia glomerata, Ochrosia apoensis, Artocarpus multifidus, Streblus elongatus, Buchanania arborescens, Greeniopsis multiflora, S. astylosa, G. rumphianum and Syzygium spp., obtained a high species importance value. The removal or loss of the species would affect the forest community. The inclusion of the Philippine endemic species, Greeniopsis multiflora and endangered species, Shorea astylosa in the list imply high priority for protection and conservation. The removal of these species with high SIV would affect the forest vegetation (Table 6).

\section{Notes on Conservation and Ecological Status}

A total of $13(5.7 \%)$ threatened species of flora were documented (see Fig. 2) based on DAO (2017) and IUCN (2018). These include six flowering plants (see Tables 2 and 3 ) and seven species of ferns and lycophytes (see Table 4). Four species are listed as critically endangered (CR), Nepenthes alfredoi V.B. Amoroso and Lagunday, Paphiopedilum ciliolare Rchb. F.) Stein., Platycerium coronarium (J. Koenig ex O.F. Mull) Desv., Shorea astylosa Foxw., Phlegmariurus phlegmaria (L.) Holub and, Sphaeropteris glauca (Blume) R.M. Tryon were listed as endangered (EN) species while five species vulnerable (VU) viz., Sphaeropteris elmeri R.M. Tryon, Lecanopteris sinuosa (Wall. Ex Hook.) Copel., Psilotum nudum (L.) Griseb., Shorea polysperma Merr., Tristaniopsis decorticata (Merr.) Peter G. Wison \& J.T. Waterh. Two species considered as Other Threatened Species (OTS), i.e. Lindsaea hamiguitanensis Karger \& V.B. Amoroso and Cinnamomum mercadoiS. Vidal.

This study, reports a total of $22(9.6 \%)$ endemic species of plants, with 16 Philippine endemic and six Mindanao endemics recorded in the expansion sites. Of these 15 species are flowering plants (see Tables 2 and 3), and 7 species are ferns and lycophytes (see Table 4). This finding implies that the flowering plants have high endemicity in the expansion sites and thus harbor species that are unique to the country.

\section{New Records of Fern and Lycophyte Species in Mt. Hamiguitan Range}

The new records of ferns and lycophytes in the Mt. Hamiguitan Range include Phlegmariurus phlegmaria (Lycopodiaceae), Pityrogramma calomelanos, (Pteridaceae) and Drynaria sparsisora (Desv.) T. Moore (Polypodiaceae).

\section{CONCLUSIONS AND RECOMMENDATION}

The botanical study in MHRWS expansion sites revealed 228 taxa of plants. Of these, 74 species were ferns and lycophytes, six species of gymnosperms, 30 species of herbs and vines and 118 species of trees and shrubs which is about $26 \%$ of the total number of plants species recorded in the entire range. Three new records of fern and lycophyte species for the entire range were documented in addition to the recent checklist increasing it to 155 . The families of trees, shrubs and other flowering plants with the highest number of species are Rubiaceae (16 species), Orchidaceae (10 species), Myrtaceae (14), Apocynaceae (7), Moraceae (6), Fabaceae (5), and the rest of the families have at most 3 species. The species diversity value for trees and pteridophytes were 1.3 and 0.6 respectively. There were 13 $(5.7 \%)$ threatened species, $22(9.6 \%)$ endemic species. Findings suggest that species in each site are unique and maybe attributed to the vegetation present, elevation variations of the different sampling sites and anthropogenic activities. The proposed expansion sites harbor diverse threatened and endemic plants deserving protection and conservation efforts. Threats to biodiversity in the sites include shifting cultivation, illegal logging and mining. Results of this study support the contention that the expansion sites, which are included in the municipal ordinances, be part of the official protected area and appeals for immediate conservation strategies by the stakeholders.

\section{ACKNOWLEDGEMENTS}

The researchers are grateful to the Biodiversity Management Bureau (BMB) and the Department of Environment and Natural Resources (DENR) of Region XI for the gratuitous permit, Central Mindanao University (CMU) administration, Center for Biodiversity Research and Extension in Mindanao (CEBREM) and Local Government Units of San Isidro, Governor Generoso and Mati for the logistics support. Most of all, sincere and utmost gratitude is conveyed to the funding agencies Commission on Higher Education (CHED) and the DENR Region XI, Davao Oriental through the active leadership of the PENRO and Park Superintendent of Mt. Hamiguitan Range Wildlife Sanctuary (MHRWS).

\section{LITERATURE CITED}

Amoroso, V.B. \& R.A. Aspiras,2011. Hamiguitan Range: a sanctuary for native flora. Saudi Journal of Biological Sciences, 18: 7-8.

Amoroso, V.B., D.A. Madulid, C.B. Amoroso, F.M. Acma, H.P Lumista, C.G. Alava, L.C. Lubos \& F.P Coritico,2011. Taxonomy and Morphology of Threatened, Rare and Economic Species (TERES) of Vascular Plants. IMDC, Central Mindanao University, 
Bukidnon, Philippines. 503 pp.

Amoroso, V.B., F.M. Acma, R.Y. Dela Cruz, F.P. Coritico, A.D. Nietes, G.B. Hamo \& H.P. Lumista,2015. Diversity of herbaceous pteridophytes in four Mindanao Long Term Ecological Research (LTER) Sites, Philippines. Asia Life Sciences, 24: 69-85.

Amoroso, V.B., F.P. Coritico \& P.W. Fritsch,2016. Species richness and conservation status of ferns and lycophytes in Mt. Hamiguitan Range Wildlife Sanctuary, Davao Oriental, Philippines. Philippine Journal of Science, 145 (2): 127-137.

Amoroso, V.B., L.D. Obsioma, J.B. Arlalejo, R.A Aspiras, D.P Capili, J.J.A. Polizon \& E.B. Sumile,2009. Inventory and conservation of endangered, endemic and economically important flora of Hamiguitan Range, Southern Philippines. Blumea, 54(1-3): 71-76.

Amoroso, V.B., R.P. Ruba, M.T. Demetillo, A. Stortelder, D.A. Lagunzad, E.C. Aranico, J.B. Arances, L.V. Rufila, P.J.A. Kessler, B.C. Tan, B.A. Roscom, N.D. Gorne, G.S. Opiso, A. Van Den Berg, W. Sm. Gruezo, L.L. Co \& C.G. Alava,2006. Plant Diversity in the Northern Landscape of Mt. Malindang Range and Environs, Misamis Occidental, Philippines. SEAMEO SEARCA. PDM Press, College Laguna, Quezon City, Philippines. ISBN: 971-560-126X. $116 \mathrm{pp}$.

Andas, S.T.,2015. Diversity of trees and pteridophytes in Pasonanca Natural Park, Zamboanga City. [Dissertation]. Musuan, Bukidnon, Philippines: Central Mindanao University. 326 pp.

Barcelona, J.F., N.E. Dolotina, G.S. Madroñero, W.G. Granert \& D.D Sopot,2006. The ferns and fern allies of the karst forests of Bohol Island, Philippines. American Fern Journal, 96: 1-20.

Barcelona, J.F.,2003. Preliminary report on the ferns and fern allies (pteridophytes) of Mt. Bali-it, Balbalasang-Balbalan National Park, Kalinga, northern Luzon, Philippines. Sylvatrop: The Technical Journal of Philippine Ecosystems and Natural Resources, 13(1 \& 2): 81-92.

Barcelona, J.F.,2004. Collection and conservation status of pteridophytes in Panay Island, Philippines. Philippine Scientist, 41: 57-73.

Belonias, B.S. \& L.M. Banoc,1994. Species diversity and distribution of pteridophytes in Mt. Pangasugan. Annals of Tropical Research, 16: 30-38.

Catapang, M.V.L., P.J.D. Reyes \& M.P. Medecilo,2012. Factors influencing species diversity of ferns in Mt. Makulot, Cuenca, Southern Luzon, Philippines. IPCBEE, 35: 98-102.

Iwatsuki, K. \& M.G Price,1977. The pteridophytes of Mt. Burnay and vicinity, northern Luzon. Southeast Asian Studies, 14: 540-572.

Myers, N., R.A. Mittermeier, C.G. Mittermeier, G.A.B. Fonseca \& J. Kent,2000. Biodiversity hotspots for conservation priorities. NATURE, 403.

Ong, P., L.E. Afuang \& R.G. Rosell-Ambal (ed.),2002. Philippine Biodiversity Conservation priorities: A Second Iteration of the National Biodiversity Strategy and Action Plan. Philippine Department of the Environmental and Natural Resources, Quezon City, Philippines. pp. 18, 113.

Pelser, P.B., J.F. Barcelona \& D.L. Nickrent (ed.),2011 onwards. Co's Digital Flora of the Philippines. www.philippineplants.org.

Salgado, E.A.,1990. A checklist of Philippine ferns. Philippine Journal of Science, 119: 107-148.

Sumagaysay C.J.L.,2012. Pteridophyte diversity of Mount Pinamantawan, Tangkulang Range, Quezon, Bukidnon. [M.Sci. Thesis]. Musuan, Bukidnon, Philippines: Central Mindanao University. 198 pp.

[DAO] DENR Administrative Order No. 2017-11,2017. Updated national list of threatened Philippine plants and their categories. http://www.denr.gov.ph.

[IUCN] International Union for Conservation of Nature,2018. The IUCN Red List of Threatened Species. Version 2018 -1. http://www.iucnredlist.org.

[UNESCO] United Nations Educational, Scientific and Cultural Organization,2014. Nine new sites inscribed on World Heritage List. Retrieved 2018-01-05. http:// whc.unesco.org 